\title{
The Danish Head and Neck Cancer database
}

This article was published in the following Dove Press journal:

Clinical Epidemiology

25 October 2016

Number of times this article has been viewed

Jens Overgaard'

Aleksandar Jovanovic ${ }^{1}$

Christian Godballe ${ }^{2,3}$

Jesper Grau Eriksen ${ }^{3}$

'Department of Experimental Clinical Oncology, Aarhus University Hospital, Aarhus, ${ }^{2}$ Department of ORL - Head and Neck Surgery, ${ }^{3}$ Department of Oncology, Odense University Hospital, Odense, Denmark
Correspondence: Jens Overgaard Department of Experimental Clinical Oncology, Aarhus University Hospital, Nørrebrogade 44, Building 5, DK-8000

Aarhus C, Denmark

Tel +4578462629

Fax +4586197109

Email jens@oncology.au.dk
Aim of the database: The Danish Head and Neck Cancer database is a nationwide clinical quality database that contains prospective data collected since the early 1960s. The overall aim of this study was to describe the outcome of the national strategy for multidisciplinary treatment of head and neck cancer in Denmark and to create a basis for clinical trials.

Study population: The study population consisted of all Danish patients referred for treatment of squamous cell carcinoma of the larynx, pharynx, oral cavity, or neck nodes from unknown primary or any histopathological type (except lymphoma) of cancer in the nasal sinuses, salivary glands, or thyroid gland (corresponding to the International Classification of Diseases, tenth revision, classifications C.01-C.11, C.30-C.32, C.73, and C.80).

Main variables: The main variables used in the study were symptoms and the duration of the symptoms; etiological factors; pretreatment and diagnostic evaluation, including tumor-nodemetastasis classification, imaging, histopathology, and laboratory tests; primary treatment with semidetailed information of radiotherapy, surgery, and medical treatment; follow-up registration of tumor status and side effects; registration of relapse and treatment thereof; and registration of death and cause of death.

Main results: Data from $>33,000$ patients have been recorded during a period of $>45$ years. In this period, the outcome of treatment improved substantially, partly due to better treatment as a result of a series of continuous clinical trials and subsequent implementation in national guidelines. The database has furthermore been used to describe the effect of reduced waiting time, changed epidemiology, and influence of comorbidity and socioeconomic parameters.

Conclusion: Half a century of registration of head and neck cancer treatment and outcome has created the basis for understanding and has substantially contributed to improve the treatment of head and neck cancer at both national and international levels.

Keywords: head and neck cancer, squamous cell carcinoma, radiotherapy, national population cohort

\section{About the Danish Head and Neck Cancer group}

The Danish Head and Neck Cancer (DAHANCA) group was established in 1976 as a working group by the Danish Society for Head and Neck Oncology with the primary aim to develop national guidelines for the treatment of head and neck cancer in Denmark. DAHANCA group's activities have included creating and running a national database, development of treatment guidelines, performing numerous national clinical protocol studies $(>30)$, and providing quality assurance programs. The group is multidisciplinary and consists of representatives from all the oncology centers in Denmark treating head and neck cancer, including oncologists, otolaryngologists, pathologists, 
imaging diagnosticians, experimental researchers, epidemiologists, and other expert support, eg, medical physicists. The DAHANCA model was behind the establishment of the structure of the Danish Multidisciplinary Cancer Groups (DMCG), and the DAHANCA group is thus the oldest of the still active DMCG.

There are $\sim 1,400$ new cases of head and neck cancer every year. Traditionally, these include cancer of the larynx, pharynx, oral cavity, sinuses, salivary glands, thyroid glands, and neck nodes from unknown primary type of cancer (corresponding to the International Classification of Diseases, tenth revision, classifications C.01-C.11, C.30-C.32, C.73, and C.80), but did not include cancer of the lip. The most significant types are cancer of the pharynx, larynx, and oral cavity. Almost all of these are squamous cell carcinomas, which etiologically are strongly linked to tobacco and alcohol use and increasingly also to infections with especially human papilloma virus, since human papilloma virus-related oropharyngeal cancer has become the most rapidly increasing cancer type in Denmark. The disease is characterized as being locoregional with late and infrequent occurring distant metastases. The therapeutic strategy is therefore aimed toward eradicating the disease at the site of the primary tumor and the regional lymph nodes. Such a treatment is typically surgery or radiotherapy, and over the past years in Denmark, focus has deliberately been on radiotherapy as the primary modality, as this ensures the best possibility of organ conservation.

DAHANCA group has been an active and well-established structure for 40 years and has provided a solid foundation for the treatment of head and neck cancer in Denmark and has become recognized internationally as one of the major head and neck cancer groups. In this aspect, it should be noted that the treatment of head and neck cancer in Denmark has rather a good outcome seen with international eyes.

\section{DAHANCA database}

The registration of patients with head and neck cancer is the essential backbone of the DAHANCA structure, and the associated database includes currently $>33,000$ patients, with an annual increase of 1,400 patients (Table 1).

Historically, the database started as a local home-designed database at the Department of Oncology, Aarhus University Hospital, Aarhus, Denmark, where the medical physicist Mogens Hjelm Hansen in the early 1970s created an elaborate program for the registration of head and neck cancer (the radiation physics department was among the first places where computers were introduced in health care). This program later formed the basis for equivalent databases in Copenhagen and Odense and a subsequent retrospectively registration of, especially, laryngeal cancer back to the early 1960s. The registration of cases later expanded to include the new oncology centers in Aalborg and Herlev, and a full national registration of larynx cancer has taken place since 1971. In the same time period, there have also been regional and more sporadic registrations of pharynx and oral cavity cancers. At a national level, the three initial databases included all patients with larynx cancer between 1971 and 1991 .

In connection with a large clinical trial, ${ }^{1}$ the national database was reconstructed and expanded to include all cancers of the larynx, pharynx, and oral cavity. Each of the five oncology centers had their own databases (named DA 1-5), which were all copies of a central master. Thus, data were gathered locally and were immediately available for daily use and research purposes at the individual institutions. The DA 1-5 data files were regularly merged into the joint database at the secretariat in Aarhus and thus formed the national DAHANCA clinical database. The structure was based on the "MedLog" program. Also, the initial three databases named (AKH, HLM, and HL3) were merged and restructured using the same program. The registration in DA 1-5 covered the period 1992-1999. In 1995, a special DATHYRCA database for registering thyroid cancer ${ }^{2}$ and more lately databases for unknown primary neck tumor, ${ }^{3}$ salivary gland tumor, ${ }^{4}$ and sinonasal cancer were established. ${ }^{5}$

In 2000, the DAHANCA database was changed into a central web-based database (OCX), which allowed interactive registration directly on a central server with the immediate possibility for quality checks of the data and with instant correction of errors. This increased the quality assurance and gave direct access to information about the current status of the patients. The OCX database is based on the development tool Clarion and uses Hypertext Transfer Protocol Secure certificate with an Hypertext Transfer Protocol Secure connection; all communications are securely encrypted. All users' logins are logged and registered. The operating system is on a Windows Server 2012 platform, and the server is situated at Aarhus University. The system is flexible and can be modified in connection with specific protocols and projects. All projects are carried out in accordance with the applicable legislation and regulations, including the European Union's Clinical Trials Directive, and corresponding regulations for the use of good clinical practice and quality assurance, and the database fulfills the Danish Data Protection Agency's description of standard specification and requirements for security. The OCX database has been active since 2000, and the structure has formed the basis for several other clinical 
Table I Number of patients included in the DAHANCA database as a function of time period and tumor site

\begin{tabular}{|c|c|c|c|c|c|}
\hline \multirow[t]{2}{*}{ Tumor site } & \multicolumn{4}{|c|}{ Time period and database acronym } & \multirow[t]{2}{*}{ All included } \\
\hline & $\begin{array}{l}\text { Pre I97 I } \\
\text { (AKH, HLM, HL3) }\end{array}$ & $\begin{array}{l}\text { I97 I-199| } \\
\text { (AKH, HLM, HL3) }\end{array}$ & $\begin{array}{l}1992-1999 \\
\text { (DA I-5) }\end{array}$ & $\begin{array}{l}2000-2015 \\
(O C X)\end{array}$ & \\
\hline Larynx & 857 & 4,092 & 2,018 & 3,694 & $|0,66|(\mathbf{9 , 8 0 4 )}$ \\
\hline Pharynx & 254 & 2,197 & $\mathrm{I}, 756$ & 6,304 & $|0,5| I(8,060)$ \\
\hline Oral cavity & & & 1,697 & 3,867 & $5,664 \quad(5,664)$ \\
\hline Sino-nasal & 114 & 276 & & 512 & $(5 \mid 2)$ \\
\hline Salivary glands & & 189 & 661 & 643 & $\mathrm{I}, 493 \quad(1,304)$ \\
\hline Thyroid & & & 652 & 3,692 & $4,344 \quad(4,344)$ \\
\hline Unknown primary & & & & 293 & $293 \quad(293)$ \\
\hline All & 1,225 & 6,754 & 6,784 & 19,005 & $33,768(29,88 I)$ \\
\hline
\end{tabular}

Note: Numbers in bold indicate the time periods with full national coverage of patient cohorts.

Abbreviation: DAHANCA, Danish Head and Neck Cancer.

quality cancer databases (eg, melanoma, sarcoma, ocular cancer, childhood cancer databases). Moreover, in 2011, the DAHANCA database was transformed from being a "private" research database to a clinical quality database in public auspices under the Danish Clinical Registries (RKKP), while still allowing the continued use for research purposes. This simplified the correlation with other public databases, such as the Danish Cancer Registry (DCR), and allowed the database to be used as an indicator of quality.

\section{Structure of the database}

Approximately 33,000 patients are included in the database, which has $\sim 300$ basic variables. ${ }^{6,7}$ Additionally, there is the possibility of specially registered parameters in connection with specific projects. The aim is that established variables remain in the database, and to have, to a large extent, continuous variables that remain unchanged. International standardized classifications are used in this context where such classifications exist (eg, Union for International Cancer Control tumor-node-metastasis classification). With regard to side effects, international principles are also applied, but the principles that we initially developed have now been adopted by other organizations, and thus, the DAHANCA database has contributed to the international standard with regard to registration of side effects from radiotherapy. As not all variables are in use at any time, the daily registration probably involves 100-150 different parameters.

The basic registration includes the following (refer forms at www.dahanca.dk):

1. On study: symptoms and the duration of the symptoms, etiological factors, pretreatment and diagnostic evaluation, including tumor-node-metastasis classification, imaging, histopathology, and laboratory tests,

2. Primary treatment: semi-detailed information of radiotherapy, surgery, and medical treatment.
3. Follow-up: registration of vital and tumor status and acute and late side effects.

4. Recurrence: registration of relapse and treatment thereof.

5. Death form: registration of death and cause of death.

Besides comparison with the DCR, vital status is checked regularly through updates from the civil registration number register. In certain situations, there have been correlations with specific extracts from the National Patient Discharge Registry and the Cause of Death Register.

\section{How do we register data?}

The principle of the DAHANCA database has throughout the years been a uniform structure of data description and a local registration. For the first many years, no actual clinical record forms existed, but the patient's journal was considered to be the paper-related data documentation and was used as a basis for data entry. For practical reasons, paper registration forms have been used since 1991 (refer forms at www.dahanca.dk). The gathering and recording of data has always been done by a few knowledgeable people, mainly senior specialist doctors. Owing to the limited amount of such people, who have been involved over many years and who know the problems in detail, both communication and collection of data are done in a very uncomplicated and pragmatic fashion. This dependency on a few highly specialized persons is both the strength and weakness of the database, because it not only gives it extremely high quality but also makes it vulnerable to changes. The database has a number of error indicators built in, which safeguards against logical errors and at the same time has the option of reminders if information is missing. Furthermore, lists of irregularities are instantly produced in a continuous attempt to collect missing data or correct any errors. 


\section{Quality assurance and correlation with other registers}

The database is verified through the numerous related research projects. ${ }^{6,7}$ Simultaneously, a specific comparison to the DCR is performed at least annually, where all cases in the DCR have been sought to be verified through the DAHANCA database, and in case of errors (in the DCR), these are explored when possible. Thus, there is currently a full national registration of DAHANCA database ranging back to 1971 (Table 1). This has shown that the DAHANCA database contains $99.7 \%$ of the originally known cases in the DCR, and an additional number of patients, currently $2 \%$, have been identified, who were not in the DCR. Furthermore, a number of erroneous registrations in the DCR have been corrected (in the DAHANCA database). The complete interaction between the two databases clearly shows that, in principle, there exists a total nationwide coverage in the DAHANCA database and further ascertains that the DAHANCA database has the potential of increasing the quality of the DCR's data. The practical aspects of such data feedback to DCR are currently being explored.

This investigative high-quality work has been performed in great detail, and in cases of doubt, relevant additional hospital records and/or other material have been obtained. Thus, all patients in the database have had their hospital records scrutinized at least once, and all updates are verified by a specialist doctor. With the DCR's well-known almost complete coverage of cancer cases in Denmark as a starting point, it is our impression that with the DAHANCA database we have a near-perfect clinical register, which probably is the world's most accurate recording of head and neck cancer treatment.

\section{Approval of the database}

The initial Aarhus-derived database was approved with register regulations at Vejle County. Later, the national database was registered and approved as a research database by the Danish Data Protection Agency (record nos 1995-1200-282 and 2004-41-4802). Since 2011, it has been a public clinical quality database (record no 1-16-02-249-14). Jens Overgaard is the overall data responsible head, and regionally, the responsibility lies with the consultant responsible for the head and neck cancer treatment at the respective oncological centers.

\section{Economy of the database}

As with other research-based databases, the project has initially been based on "con amore" activities performed by interested professionals at weekends and in late hours. Gradually, the need for organized help has increased and the database has throughout the years been partly financed in connection with research projects, where among others, $\mathrm{PhD}$ students were assigned to the activities mainly sponsored through the Danish Cancer Society and the Medical Research Council (we have constantly tried to avoid any commercial influence and sponsoring). Since 2006, we have been partly supported thorough the DMCG/RKKP structure. However, the activities have throughout benefited from being part of the infrastructure at the Department of Experimental Clinical Oncology at Aarhus University Hospital and from the numerous dedicated persons who have used their spare time to strengthen the database.

\section{Use of the database}

The value of any database is linked with its use, and the quality data are accessible through access to the RKKP (http:// www.rkkp.dk/forskningsadgang/). Throughout the years, the DAHANCA database has been extensively explored and has served as an active research database that has formed the basis for numerous scientific projects and publications, including $15 \mathrm{PhD}$ and doctoral theses (for full list, refer the DAHANCA homepage [www.dahanca.dk]). It has been the supporting database for $>30$ prospective clinical trials and has acted as a baseline for large retrospective studies describing the natural history and treatment outcome in head and neck cancer. Examples of studies ${ }^{6,8-11}$ are given in Figure 1, and more can be found on the DAHANCA web site. This means that the database is constantly revised and updated and, consequently, is extremely well verified.

Since many of the DAHANCA protocols have focused on biological-based improvement of head and neck cancer treatment, these translational studies have been based on a large amount of clinical material. ${ }^{10-13}$ This DAHANCA-related biobank contains biological material (tumor, normal tissue cell lines, blood samples, DNA, RNA, etc) from $>4,000$ patients with detailed clinical data and follow-up. Also, paraffinembedded tumor biopsies are available from almost all patients. Currently, we are systematically collecting material from new patients in the National Danish Cancer Biobank.

The database has not only been strengthened by linkage to other registries ${ }^{6,7}$ but has also shown its value by being a supportive basis for studies of comorbidity, ${ }^{6}$ value of follow-up ${ }^{14}$ or socioeconomical relationship in patients with head and neck cancer. ${ }^{15,16}$ In the foreseen future, it will be part of international comparisons of treatment strategies, and for such purpose, a clinical database is far superior than using cancer registry comparisons (such as the Nordic NORDCAN study), since such databases are incomplete and inaccurate 
A

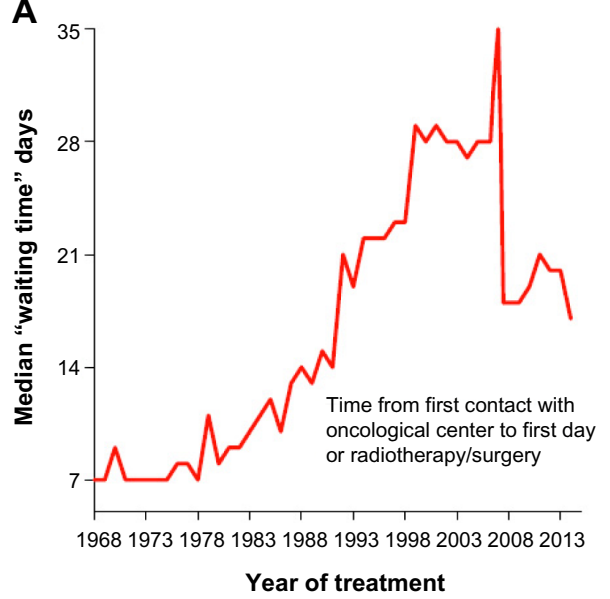

C

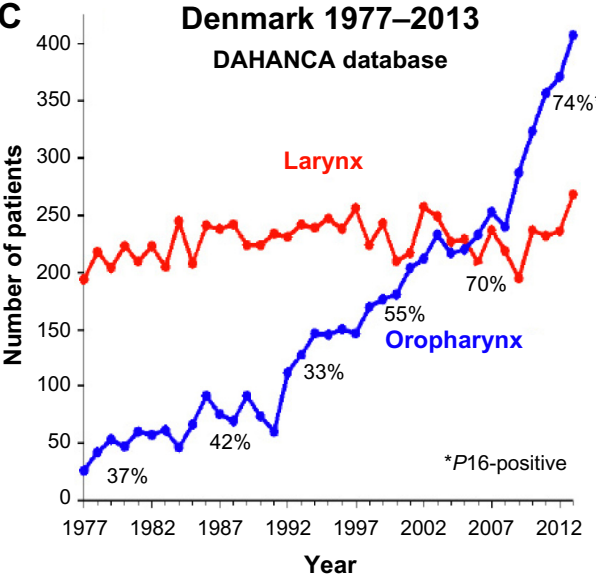

B

DAHANCA database

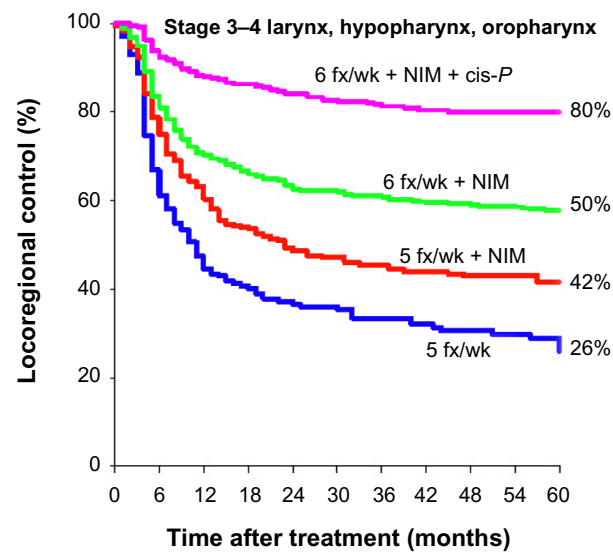

D Comorbidity and survival

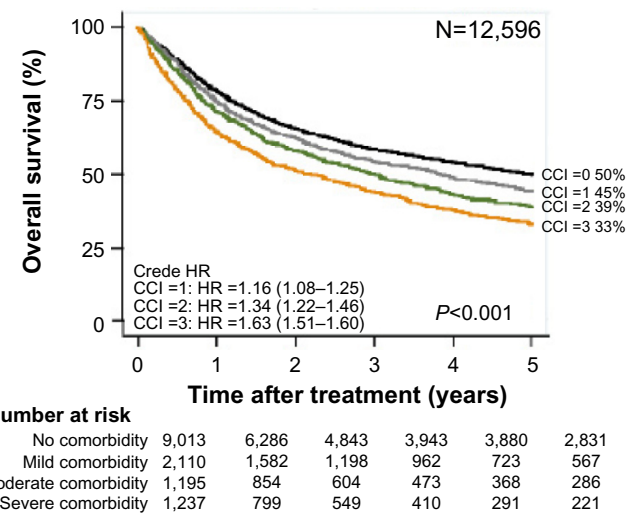

Figure I Examples of studies involving the DAHANCA database.

Notes: Panel $(\mathbf{A})$ shows the development in waiting time between diagnosis and start of treatment. The prominent increase found in the DAHANCA database was the direct cause that released a massive intervention in the form of "cancer packages"' aimed to reduce the waiting time (implemented in September 2006). Panel (B) shows an illustration of biological modification of radiotherapy seen in a series of clinical trials in the treatment of advanced larynx and pharynx carcinoma. Conventional fractionated treatment with 66-68 Gy given in five weekly fractions (fx) of 2 Gy was significantly enhanced by adding the hypoxic radiosensitizer NIM to overcome hypoxic radioresistance. This was further improved by treating with six weekly fractions and thereby reducing the overall treatment time to compensate for radiation-induced accelerated repopulation of tumor stem cells. Finally the intrinsic radioresistance was targeted by adding concomitant chemotherapy with cis-P. Overall, did such strategy lead to a threefold improvement in treatment outcome? The validity of this DAHANCA strategy is now been explored in a large clinical trial in Europe, North America, and Australia. Modified from Baumann M, Krause M, Overgaard J, et al. Radiation oncology in the era of precision medicine. Nat Cancer Rev. 20I6;16:234-249." Panel (C) shows the crude incidence of laryngeal and oropharyngeal carcinomas in Denmark during 1977-20I3 and its relationship with HvP/p I6 positivity. Reprinted from Radiother Oncol, 95 , Lassen $\mathrm{P}$, The role of human papillomavirus in head and neck cancer and the impact on radiotherapy outcome, 37I-380, Copyright (20I0), with permission from Elsevier. ${ }^{2}$ Panel (D) shows the impact of Charlson Comorbidity Index (CCI) on outcome of treatment in a cohort of I2,623 Danish HNSCC patients, I992-2008. The study resulted in creating a specific head and neck cancer comorbidity index, now implemented in routine use. Reproduced from Bøje CR, Dalton SO, Grønborg TK, et al. The impact of comorbidity on outcome in 12623 Danish head and neck cancer patients: a population based study from the DAHANCA database. Acta Oncol. 20I3;52:285-293. ${ }^{6}$

Abbreviations: DAHANCA, Danish Head and Neck Cancer; NIM, nimorazole; cis-P, cisplatin; HVP, human papilloma virus; HNSCC, head and neck squamous cell carcinoma; wk, week; HR, hazard ratio.

regarding clinical information. Thus, it is important to use the right tools/databases for the right purposes, and when conclusions about therapeutic strategies are the aim, the data must be obtained from a well-verified clinical quality database. We believe that the DAHANCA database is a prime example of such an activity.

\section{Acknowledgment}

This article was funded by the Danish Cancer Society and the Program for Clinical Research Infrastructure established by the Lundbeck Foundation and the Novo Nordisk Foundation and administered by the Danish Regions.

\section{Disclosure}

The authors report no conflicts of interest in this work.

\section{References}

1. Overgaard J, Hansen HS, Specht L, et al. Five compared with six fractions per week of conventional radiotherapy of squamous-cell carcinoma of head and neck: DAHANCA 6 and 7 randomised controlled trial. Lancet. 2003;362:933-940. 
2. Londero SC, Mathiesen JS, Krogdahl A, et al. Completeness and validity in a national clinical thyroid cancer database: DATHYRCA. Cancer Epidemiol. 2014;38:633-637.

3. Grau C, Johansen LV, Jakobsen J, Geertsen P, Andersen E, Jensen BB. Cervical lymph node metastases from unknown primary tumours. Results from a national survey by the Danish Society for Head and Neck Oncology. Radiother Oncol. 2000;55:121-129.

4. Bjørndal K, Krogdahl A, Therkildsen MH, et al. Salivary gland carcinoma in Denmark 1990-2005: a national study of incidence, site and histology. Results of the Danish Head and Neck Cancer Group (DAHANCA). Oral Oncol. 2011;47:677-682.

5. Thorup C, Sebbesen L, Danø H, et al. Carcinoma of the nasal cavity and paranasal sinuses in Denmark 1995-2004. Acta Oncol. 2010;49:389-394.

6. Bøje CR, Dalton SO, Grønborg TK, et al. The impact of comorbidity on outcome in 12623 Danish head and neck cancer patients: a population based study from the DAHANCA database. Acta Oncol. 2013;52:285-293.

7. Lyhne NM, Johansen J, Kristensen CA, et al. Incidence of and survival after glottic squamous cell carcinoma in Denmark from 1971 to 2011 a report from the DAHANCA group. Eur J Cancer. 2016;59:46-56.

8. Jensen AR, Nellemann HM, Overgaard J. Tumor progression in waiting time for radiotherapy in head and neck cancer. Radiother Oncol. 2007;84:5-10.

9. Lyhne NM, Christensen A, Alanin MC, et al. Waiting times for diagnosis and treatment of head and neck cancer in Denmark in 2010 compared to 1992 and 2002. Eur J Cancer. 2013;49:1627-1633.
10. Lassen P, Eriksen JG, Hamilton-Dutoit S, Tramm T, Alsner J, Overgaard J. Effect of HPV-associated p16INK4A expression on response to radiotherapy and survival in squamous cell carcinoma of the head and neck. J Clin Oncol. 2009;27:1992-1998.

11. Baumann M, Krause M, Overgaard J, et al. Radiation oncology in the era of precision medicine. Nat Cancer Rev. 2016;16:234-249.

12. Lassen P. The role of human papillomavirus in head and neck cancer and the impact on radiotherapy outcome. Radiother Oncol. 2010;95:371-380.

13. Toustrup K, Sørensen BS, Nordsmark M, et al. Development of a hypoxia gene expression classifier with predictive impact for hypoxic modification of radiotherapy in head and neck cancer. Cancer Res. 2011;71:5923-5931.

14. Pagh A, Vedtofte T, Lynggaard CD, et al. The value of routine followup after treatment for head and neck cancer. A national survey from DAHANCA. Acta Oncol. 2013;52:277-284.

15. Kjær T, Bøje CR, Olsen MH, et al. Affiliation to the work market after curative treatment of head-and-neck cancer: a population-based study from the DAHANCA database. Acta Oncol. 2013;52:430-439.

16. Olsen MH, Bøje CR, Kjær TK, et al. Socioeconomic position and stage at diagnosis of head and neck cancer - a nationwide study from DAHANCA. Acta Oncol. 2015;54:759-766.
Clinical Epidemiology

\section{Publish your work in this journal}

Clinical Epidemiology is an international, peer-reviewed, open access, online journal focusing on disease and drug epidemiology, identification of risk factors and screening procedures to develop optimal preventative initiatives and programs. Specific topics include: diagnosis, prognosis, treatment, screening, prevention, risk factor modification,

Submit your manuscript here: http://www.dovepress.com/clinical-epidemiology-journal

\section{Dovepress}

systematic reviews, risk \& safety of medical interventions, epidemiology \& biostatistical methods, and evaluation of guidelines, translational medicine, health policies \& economic evaluations. The manuscript management system is completely online and includes a very quick and fair peer-review system, which is all easy to use. 